\title{
Tidal stripping of stars near supermassive black holes
}

\author{
L. Dai ${ }^{\mathrm{a}}$ and R. Blandford \\ Kavli Institute for Particle Astrophysics and Cosmology, Stanford University, Menlo Park, CA 94025, USA
}

\begin{abstract}
In a binary system composed of a supermassive black hole and a star orbiting the hole in an equatorial, circular orbit, the stellar orbit will shrink due to the action of gravitational radiation, until the star fills its Roche lobe outside the Innermost Stable Circular Orbit (ISCO) of the hole or plunges into the hole. In the former case, gas will flow through the inner Lagrange point (L1) to the hole. If this tidal stripping process happens on a time scale faster than the thermal time scale but slower than the dynamical time scale, the entropy as a function of the interior mass is conserved. The star will evolve adiabatically, and, in most cases, will recede from the hole while filling its Roche lobe. We calculate how the stellar equilibrium properties change, which determines how the stellar orbital period and mass-transfer rate change through the "Roche evolution" for various types of stars in the relativistic regime. We envisage that the mass stream eventually hits the accretion disc, where it forms a hot spot orbiting the hole and may ultimately modulate the luminosity with the stellar orbital frequency. The ultimate goal is to probe the mass and spin of the hole and provide a test of general relativity in the strong-field regime from the resultant quasi-periodic signals. The observability of such a modulation is discussed along with a possible interpretation of an intermittent 1 hour period in the X-ray emission of RE J1034 + 396.
\end{abstract}

\section{INTRODUCTION}

When a star passes by a supermassive black hole $(\mathrm{SMBH})$ closely, tidal effects can be induced. There are two extreme cases. The first case is a tidal disruption event, to which most talks in the meeting were dedicated. Tidal disruption can happen when a star passes close by a black hole in an unbound orbit or a very eccentric orbit. The stellar volume exceeds the Roche volume at periastron, and the star is torn apart and eventually swallowed by the hole (e.g., [11]). As broadly discussed in the meeting, one convincing instance of a tidal disruption event is the recent luminous Sw J1644 + 57 X-ray observation $([2,15])$. Optical and UV flares have also been associated with tidal disruptions (e.g., $[7,13])$.

The other case, which was the main topic of my talk, is called tidal stripping or stable Roche masstransfer. In an Extreme Mass-Ratio Inspiral (EMRI) system composed of a central SMBH and a star, the stellar orbit shrinks due to the loss of energy and angular momentum through gravitational radiation and other torques. We restrict our discussion of EMRI systems to stars in circular, equatorial orbits. If such a star overflows its Roche lobe close to the innermost stable circular orbit (ISCO), the star loses mass adiabatically through the inner Lagrange point (L1), and the stellar orbit will usually expand (eventually). Gas flowing through L1 will generally form a torus orbiting the hole. A hot spot will be formed where the stream hits the disc. The hot spot (and shocks generated) will orbit the hole with the stellar orbital frequency, and an inclined observer should see a modulation of the radiation from the disc, most likely in the X-ray range. Clearly the modulation will be the greatest when the observer is in the equatorial plane, observing maximal Doppler boosting. This process is illustrated in Fig. 1. A tidal stripping event, not as luminous as a tidal disruption event, however, lasts much

\footnotetext{
a e-mail: cosimo@stanford.edu
}

longer. It is easier to be modeled with high precision for the mass and spin of the hole. Therefore, it could provide a probe of detecting SMBH spins and testing general relativity.

In this paper, we will only focus on the relativistic treatment of the problem as it is more intriguing. The details of Newtonian and relativistic calculations can be found in [4].

\section{RELATIVISTIC ROCHE MASS-TRANSFER}

\subsection{Gravitational inspiral of the star}

We restrict our attention to circular equatorial orbits, since the orbit circularizes quickly as it loses angular momentum and energy due to gravitational radiation. The star will plunge into the hole when its orbit shrinks to the size of the ISCO. The relativistic inspiral time for a star starting from an orbital radius $r$ till it plunges into the hole is:

$$
t_{\text {inspiral }}=\frac{5}{256} \frac{M^{2}}{m_{\star} \tilde{\Omega}^{\frac{8}{3}}} \mathcal{T}
$$

([5]) in the Boyer-Lindquist coordinate, where $M$ is the mass of the hole, $m_{\star}$ is the stellar mass, $\tilde{\Omega}$ is the dimensionless orbital angular velocity defined by $\tilde{\Omega}=$ $M \Omega$, where $\Omega$ is the regular orbital angular velocity. The relativistic Kepler's Second Law gives:

$$
\tilde{\Omega}=\frac{1}{\tilde{r}^{\frac{3}{2}} \pm a} .
$$

where \pm refers to prograde and retrograde orbits, $a$ is the spin parameter of the hole, and $\tilde{r}=\frac{r}{R_{q}}\left(R_{g}\right.$ is the gravitational radius of the hole).

$\mathcal{T}$ is the relativistic correction term. We interpolated the tabulated results of $\mathcal{T}$ in [5] to get a convenient 


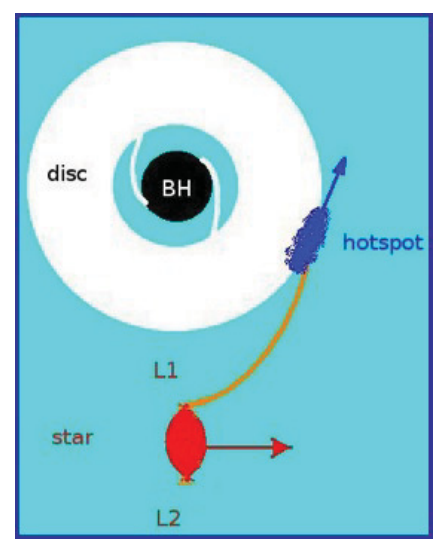

Figure 1. A star filling its Roche lobe is tidally stripped by a massive black hole.

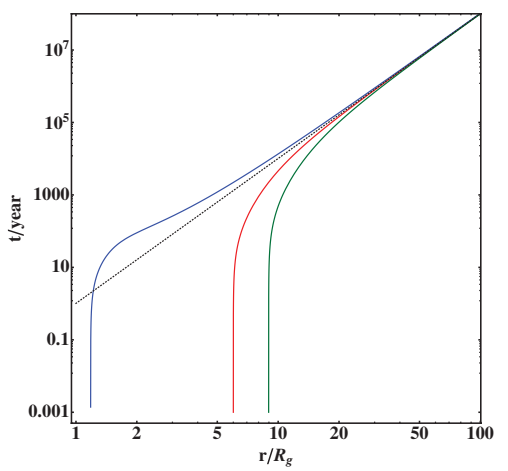

Figure 2. The total inspiral time in years as a function of $r / R_{g}$ for a $0.3 M_{\odot}$ dwarf star orbiting a $10^{7} M_{\odot}$ hole. This plot is in the log-log scale. The three colors indicate three different spin parameters of the hole: -0.99 (green), 0 (red), and 0.999 (blue). The three solid curves are accurate inspiral times obtained using Eq. (2). The black dotted curve is the Newtonian inspiral time as in [10]. All other curves approach the Newtonian curve when the star is far away from the hole.

formula:

$$
\begin{aligned}
\mathcal{T}(x, a)= & \frac{(x-1)^{2}+b(x-1)}{x^{2}+d x+f} \\
b= & 1.06 \times(1-a)^{-0.11}-0.66 \\
d= & 0.57 \times(1-a)^{0.23}-2.22 \\
f= & {\left[-1.08 \times(1-a)^{1.08}-3.18 \times(1-a)^{-0.018}\right] } \\
& \times e^{-0.23 \times(1-a)}+4.91
\end{aligned}
$$

where $x=r / r_{\text {ISCO }}$. This formula holds true with a maximum error of $\sim 2 \%$ for $x \geq 2$.

The inspiral time of $\mathrm{a} \sim 0.3 M_{\odot}$ dwarf star falling into a $10^{7} M_{\odot}$ hole is shown in Fig. 2. It is clear that the standard "Landau-Lifschitz" form equation is adequate for $r>100 R_{g}$. It is arguable that faraway stars on circular orbits can take too long to get close enough to the black hole. Therefore, other mechanisms must happen to shorten the inspiral time. Stars can be borne in the accretion disc, when materials accrete onto the black holes and finally go away eventually leaving only the stars. Such stars would orbit the hole in close, bound orbits in the equatorial plane with low eccentricity. When other stars and gas are around, dynamical frictions and three-body interactions can also drag in a faraway star.

The gravitational radiation reaction torque is readily computed from:

$$
\tau(r, a)_{\mathrm{gr}}=-\left(\frac{\partial L}{\partial r}\right)_{a} /\left(\frac{\partial t_{\text {inspiral }}}{\partial r}\right)_{a}
$$

where the orbital angular momentum is (e.g., [1])

$$
L=m_{\star} M \frac{\left(1-2 \tilde{r}^{-1}+a * \tilde{r}^{-\frac{3}{2}}\right) r^{\frac{1}{2}}}{\left(1-3 \tilde{r}^{-1}+2 a * \tilde{r}^{-\frac{3}{2}}\right)^{\frac{1}{2}}} .
$$

\subsection{Roche limit}

Tidal stripping starts if the star fills the Roche lobe outside the ISCO. The Roche lobe is an equi-potential surface of the first Lagrange point (L1), at which the gradient of the Roche potential vanishes. The Roche potential is the sum of the gravitational potentials of the star and the hole, plus the potential in the frame rotating with the star due to the centrifugal force. It can be calculated by expanding the local tidal tensor in an orthonormal coordinate basis freely falling with the star to be:

$$
\begin{aligned}
\Phi(x, y, z)= & -m_{\star}\left(x^{2}+y^{2}+z^{2}\right)^{-\frac{1}{2}} \\
& +\frac{M}{r^{3}} \frac{k y^{2}+z^{2}-(k+1) x^{2}}{2}-\frac{M}{r^{3}} \frac{k_{\omega}\left(x^{2}+y^{2}\right)}{2} .
\end{aligned}
$$

Here $\{x, y, z\}$ is a locally orthonormal coordinate system centered at the star and directed along the $\{r, \theta, \phi\}$ coordinate axes. $k_{\omega}$ is the stellar rotational parameter in the inertial frame, ranging between 0 (non-rotating star) and 1 (co-rotating star), and $k$ is a parameter defining how relativistic the motion is:

$$
k=\frac{\tilde{r}^{2}-4 a \tilde{r}^{\frac{1}{2}}+3 a^{2}}{\tilde{r}^{2}-3 \tilde{r}+2 a \tilde{r}^{\frac{1}{2}}} .
$$

Using this potential, an adequate approximation to the Roche volume is:

$$
V\left(r, a, k_{\omega}\right)=V_{\mathrm{N}}\left(r, k_{\omega}\right)-\frac{V_{\mathrm{N}}\left(r, k_{\omega}\right)-V_{\mathrm{ISCO}}\left(r, k_{\omega}\right)}{\left(\frac{r}{r_{\text {ISCO }}}\right)^{0.5}+F\left(a, k_{\omega}\right)\left(\frac{r}{r_{\text {ISCO }}}-1\right)},
$$

where

$$
\begin{aligned}
F\left(a, k_{\omega}\right)= & -23.3+\frac{13.9}{k_{\omega}+2.8}+\left(23.8-\frac{14.8}{2.8+k_{\omega}}\right) \\
& \times(1-a)^{0.02}+\left(0.9-\frac{0.4}{2.6+k_{\omega}}\right) \times(1-a)^{-0.16} .
\end{aligned}
$$

$V_{N}$ is the Newtonian Roche volume:

$$
V_{N} \simeq 0.683 \times\left(\frac{k_{\omega}}{2.78}+1\right)^{-1} \frac{m_{\star}}{M} r^{3} .
$$

And $V_{\text {ISCO }}$ is the Roche volume at the ISCO $(\mathrm{k}=2)$ :

$$
V_{\text {ISCO }}=0.456 \times\left(\frac{k_{\omega}}{4.09}+1\right)^{-1} \frac{m_{\star}}{M} r^{3} .
$$


In summary, the relativistic volume has a similar form as the Newtonian formula - a constant times the ratio of the two masses times the distance between the two objects. The constant, ranging between 0.368 and 0.683 , depends on $k$ (how relativistic the motion is) and $k_{\omega}$ (how fast the star rotates in inertial frames). Comparing with Fishbone's Roche volume model ([6]) used by Hameury ([9]), which didn't consider the black hole spin and assumed the star to be co-rotating, our Roche volume could be $20-40 \%$ different in value as the star gets close to the ISCO. We will show that it is crucial to give this problem a full relativistic treatment in Section 2.4.

\subsection{Adiabatic evolution of the star}

We used real stellar models kindly provided by Peter Eggleton to study how stars respond to the loss of mass adiabatically on a time scale slower than the dynamical time scale but still faster than the thermal one. The stellar orbital radius from the hole changes as mass is lost. The way that the star evolves in such a mass-transfer environment depends mainly upon the mean density of the stripped star as a function of decreasing mass. Nuclear reactions will be shut off as soon as the mass loss starts and the central temperature decreases. For details please refer to [3].

As the star is stripped on an adiabatic time scale, its entropy as a function of interior mass is conserved. For a star composed of ideal gas, ignoring radiative contributions, Coulomb and degeneracy corrections, its equilibrium properties satisfy:

$$
\begin{aligned}
\frac{d r(m)}{d m} & =\frac{\tilde{S}(m)}{4 \pi r(m)^{2} p(m)^{\frac{3}{5}}}, \\
\frac{d p(m)}{d m} & =-\frac{G m}{4 \pi r(m)^{4}} .
\end{aligned}
$$

Here $m$ is the interior mass, $r(m)$ is the stellar radius as a function of $m, p(m)$ is the interior gas pressure, $G$ is the gravitational constant, $\rho(m)$ is the stellar density. $\tilde{S}=p^{3 / 5} \rho^{-1}$ is the effective entropy, which is a known function of $m$ using our stellar models. Therefore, we can use these equations to solve for the stellar properties as its total mass decreases.

We considered the adiabatic evolution of not only main-sequence stars but also a representative set of stars and planets under these conditions. We found that as mass is lost, the pressure will decrease, gas will expand, but the total stellar volume might increase or decrease. As a result, stellar mean density might increase or decrease. We found that lower main-sequence stars, brown dwarfs, white dwarfs, and Jupiter-like planets will have their mean densities decrease all the time as mass is stripped. Upper main-sequence stars, Sun-like stars, red giants will have their mean densities increase and then decrease through the mass-loss.

\subsection{Stellar orbit evolution and Roche mass-transfer}

Using the relativistic Kepler's Second Law (Eq. (2)), and the results of relativist Roche Volume (Eq. (8)), we obtain

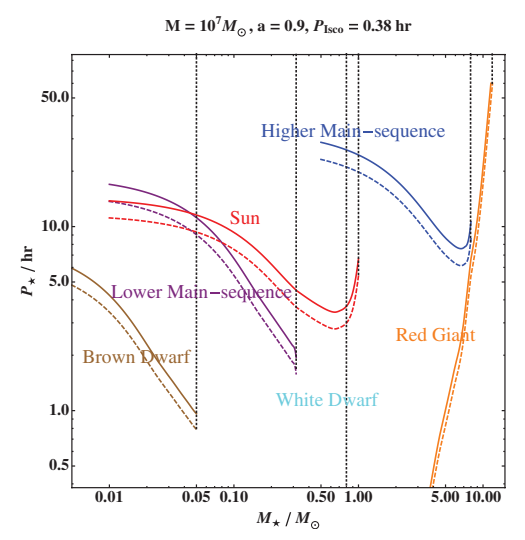

Figure 3. Roche evolutions of different stars close to SMBHs with $M=10^{7} M_{\odot}$ and $a=0.9$. The x-axis is the stellar mass, and the y-axis is the stellar orbital period from the ISCO to $100 R_{g}$. The solid line are the relativistic calculations for co-rotating stars, and the dashed lines are the relativistic calculations for nonrotating stars. The vertical dotted lines represent the phase of inspiral due to gravitational radiation.

a simple relationship between the stellar mean density and the orbital radius when the star fills its Roche lobe:

$$
\begin{aligned}
\frac{M}{\bar{\rho}_{\star} r^{3}}= & -\frac{0.683 \times\left(\frac{k_{\omega}}{2.78}+1\right)^{-1}-0.456 \times\left(\frac{k_{\omega}}{4.09}+1\right)^{-1}}{\left(\frac{r}{r_{\mathrm{ISCO}}}\right)^{0.5}+F\left(a, k_{\omega}\right)\left(\frac{r}{r_{\mathrm{ISCO}}}-1\right)} \\
& +0.683 \times\left(\frac{k_{\omega}}{2.78}+1\right)^{-1} .
\end{aligned}
$$

As a star approaches a SMBH in a circular, equatorial orbit, it might plunge into the SMBH directly, or go through full Roche evolution, or start the Roche evolution then plunge into the hole. The detailed evolution depends on the mass and spin of the black hole, the stellar type, and the rotational parameter $k_{\omega}$ of the star. Using Eq. (12), we can check when the star will start Roche accretion and if so, how its orbit will change through the process. We summarized the inspiral/outspiral scenarios for different stars and hole parameters in Fig. 9 in our paper [4]. For example, Fig. 3 shows how different stars behave when approaching a hole with $M=10^{7} M_{\odot}$ and $a=0.9$ on circular, equatorial orbits: A white dwarf will plunge in directly. A brown dwarf and a lower main-sequence star will spiral out since mass-transfer starts. A higher main-sequence star and a Sun-like star will spiral in first then eventually spiral out. A red super giant star, with its envelop being stripped, will keep on spiraling in till its core plunges into the hole. The qualitative behavior of the orbital evolution is similar using Newtonian or relativistic models for non-rotating or co-rotating stars, with some quantitative differences.

From the conservation of angular momentum, the stellar angular momentum $L$ satisfies:

$$
\Delta L=\Delta L_{\mathrm{GR}}+\Delta L_{\mathrm{ISCO}}
$$

Here $L$ is the angular momentum of the star as in Eq. (5). $\Delta L_{\mathrm{GR}}$ is caused by the gravitational radiation torque $\tau_{\mathrm{gr}}$ calculated from our new model (Eq. (4)). $\Delta L_{\mathrm{ISCO}}$ represents the loss of angular momentum of materials falling into the 


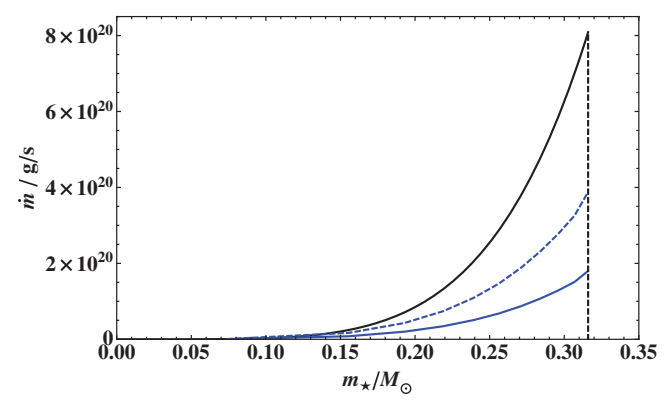

Figure 4. This figure shows how fast a non-rotating lower mainsequence star with initial mass $\sim 0.3 M_{\odot}$ is tidally stripped by a Schwarzschild SMBH with a mass $10^{7} M_{\odot}$. The x-axis is the stellar mass, and the y-axis is the mass-loss rate. The black curve is the Newtonian calculation, the solid blue curve is the relativistic calculation, and the dashed blue curve is the relativistic calculations ignoring the angular momentum loss at the ISCO.

ISCO. For simplicity we ignored the disc radiation and disc wind. We then have:

$$
\frac{d L}{d t}+\tau_{\mathrm{gr}}+\dot{m}_{\star} j_{\mathrm{ms}}=0 .
$$

$\dot{m}_{\star}$ is the stellar mass-loss rate, and $j_{\mathrm{ms}}$ is the specific angular momentum at the ISCO, which equals 0 in the Newtonian limit.

For example, we can calculate how a dwarf star with mass $\sim 0.3 M_{\odot}$ loses mass at different rate in relativistic and Newtonian models. With general relativity considered, the star does not recede from the hole as far as in the Newtonian limit (as in Fig. 3), and the mass accretion rate drops by about half (as in Fig. 4).

In order to see if this Roche mass-transfer is dynamically stable, as Hameury ([9]) suggested, we can check whether the star will exceed the Roche surface under the assumption of no angular momentum loss through gravitational radiation. Hameury's model suggests that such Roche mass-transfer can be dynamically unstable for some stars, e.g. a lower-main sequence star around a $10^{7} M_{\odot}$ hole. However, using our more accurate relativistic models, we found that all the stars go through tidal stripped dynamically stably, in contradiction to Hameury's results.

\section{OBSERVATION OF RE J1034 + 396 AND FUTURE DIRECTIONS}

One observation that may possibly be explained by this model is the quasi-periodic XMM-Newton $\mathrm{X}$-ray signal from a nearby active Seyfert I galaxy RE $\mathrm{J} 1034+396$ ([8]). As shown in Fig. 1 in their paper, the rest-frame X-ray emission has a periodicity of $\sim 1 \mathrm{hr}$. The $\mathrm{X}$-ray flux has a modulation of $\sim 0.1$ associated with the nucleus of the galaxy, and this modulation could be well fitted by a cosine function. Using our model, a dwarf star or brown dwarf or red giant can produce emissions with such a periodicity when tidally stripped by a $\sim 10^{6}-10^{7} M_{\odot}$ $\mathrm{SMBH}$, though the mass accretion rate under adiabatic condition and pure gravitational radiation torque is too low to produce the observed X-ray flux. 16 cycles of signals are insufficient to be confident of a stable period. However, if more signals can be observed, information on spin could be extracted. In principle, the closer the star is to the hole, or the larger the spin of the hole, the stronger the emission would be. This could introduce a selection effect as rapidly spinning holes would be more likely to be observed.

We also simulated periodic signals from a hot spot orbiting around the hole circularly and equatorially using our numerical package. The modulation of intensity comes from beaming effect. The shape of the curves and the relative intensity are different for different spins and observer inclination angles, from which we can probe the parameters of the hole. Also our model is different from other quasi-periodic oscillation (QPO) models (e.g. [14]) in producing a hot spot on the disc, as the periodicity of the modulation will follow that of the stellar orbit although there is likely to be phase noise. Since the stellar orbit evolves on an adiabatic time scale, the periodicity of the hot spot will change slowly but in a consistent way. For example, an accreting $\sim 0.3 M_{\odot}$ dwarf star's orbital periodicity can change by $\sim 0.1 \mathrm{~s}$ per year, during which we can observe $\sim 8000$ cycles of modulation. A $P-\dot{P}$ analysis similar to the double pulsar's could in principle be done to extract information of the stellar type and black hole parameters.

Interesting future projects include: (1) calculating the probability of observing tidal stripping events by studying stellar distributions in the cusps near the hole and the mechanisms of bringing stars close to the ISCO; (2) analyzing the radiation mechanism of the hot spot produced by gas stream hitting the disc; (3) investigating the final fate of a star as it migrates outward after the onset of mass- transfer; and most importantly, (4) searching existing X-ray databases, to seek evidence for stars in bound orbits around SMBHs and QPOs associated with SMBHs.

\section{References}

[1] Bardeen J. M., Press W. H., Teukolsky S. A., 1972, APJ, 178, 347

[2] Bloom J. S., Giannios D., Metzger B. D., et al. 2011, Science, 333, 203

[3] Dai L., Blandford R. D., P. P. Eggleton, 2011, ArXiv e-prints

[4] Dai L., Blandford R. D., 2011, ArXiv e-prints

[5] Finn L. S., Thorne K. S., 2000, Physical Review D, 62, 124021

[6] Fishbone L. G., 1973, APJ, 185, 43

[7] Gezari S., Chornock R., Rest A., et al. 2012, Nature, 485, 217

[8] Gierliński M., Middleton M., Ward M., Done C., 2008, Nature, 455, 369

[9] Hameury J., King A. R., Lasota J., Auvergne M., 1994, Astronomy and Astrophysics, 292, 404

[10] Misner C. W., Thorne K. S., Wheeler J. A., 1973, Gravitation. San Francisco: W.H. Freeman and Co., 1973

[11] Rees M. J., 1988, Nature, 333, 523 
[12] Reynolds C. S., Nowak M. A., Phys. Rep., 2003, 377, 389

[13] van Velzen S., Farrar G. R., Gezari S., Morrell N., Zaritsky D., Ostman L., Smith M., Gelfand J., Drake A. J., 2011, APJ, 741, 73
[14] Wagoner R. V., Silbergleit A. S., OrtegaRodríguez M., 2001, APJ, Part 2 - Letters, 559, L25

[15] Zauderer B. A., Berger E., Soderberg A. M., Loeb A., Narayan R., et al. 2011, Nature, 476, 425 